\title{
A Study on the Effect of a 10-Degree Rotating Sonic Toothbrush on Oral Hygiene Management
}

\author{
Jin-Sil Kim ${ }^{1}$, Chung-jae Lee ${ }^{2}$ \\ ${ }^{1}$ Department of Dental Hygiene, Busan Women's College, Busan, ${ }^{2}$ Department of Dental Technology \& Science, Shinhan \\ University, Uijeongbu, Korea
}

\begin{abstract}
Objective: This study excluding the use of auxiliary oral products such as dental floss and interdental toothbrushes, which are effective in managing the dental plaque between teeth, a manual toothbrush and a sonic vibrating toothbrush was used for the experimental group to clinically compare the effectiveness of removing plaque from the teeth to help in choosing toothbrushes.
\end{abstract}

Methods: A total of 60 volunteers with early gingivitis in their 30s or older who voluntarily agreed to this study were included. In the oral examination, the Simple Patient Hygiene Performance (S-PHP) index, gingivitis index, and halitosis were measured, and the values were recorded before the experiment, 1st week of the experiment, 2nd week of the experiment, and 4th week of the experiment.

Results: It was found that there was an interaction effect between the two groups according to the period of toothbrush use. It was confirmed that the period of toothbrush use had an effect on the S-PHP index. The gingivitis index of the group using the toothbrush of the experimental group was found to decrease statistically significantly over the period of toothbrush use. An interaction effect between the two groups according to the period of toothbrush use.

Conclusion: The S-PHP index of the group using the experimental group toothbrush showed a statistically significant decrease over the period of toothbrush use $(p<0.001)$. As a result of analyzing the change in the gingivitis index, it was found that the gingivitis index of the group using the experimental group toothbrush decreased statistically. The concentration of halitosis in the group using the toothbrush of the experimental group was found to decrease statistically significantly over the period of toothbrush use $(\mathrm{p}<0.001)$.

Keywords: interdental toothbrushes, halitosis measurement, rotating sonic toothbrush

Corresponding author Chung-jae Lee

E-mail: best8463@hanmail.net

(iD) https://orcid.org/0000-0001-5648-0830

Received December 26, 2021, Revised December 31, 2021, Accepted December 31, 2021

\section{Introduction}

Accumulated dental plaque increases the tendency for gingival redness, edema, and gingival bleeding. Therefore, oral hygiene is essential for the prevention of gingivitis and periodontal disease, and gingivitis can be prevented by managing the dental plaque through oral hygiene education and receiving scaling from the dentist [1,2].

Copyright (C) 2021. Korean Academy of Preventive Dentistry.

This is an Open Access article distributed under the terms of the Creative Commons Attribution Non-Commercial License (http://creativecommons.org/licenses/ by-nc/4.0) which permits unrestricted non-commercial use, distribution, and reproduction in any medium, provided the original work is properly cited. 
Tooth brushing to remove dental plaque removes unstably formed tartar and smoothens the tooth surface to clean the tooth surface to suppress the re-adhesion of food residues or the re-formation of the dental plaque. So, it is important to brush in the right way for a sufficient amount of time.

Toothbrushes have a wide variety of shapes, and the size and shape of the head of the toothbrush, the method of operation, the size of the length of the handle [3], the inclination of the handle, and the head vary. In general, the method of maintaining cleanliness using a manual toothbrush is common, but the function of electric toothbrushes that can easily brush teeth by reproducing the brushing technique without effort has been proven, thereby increasing the demand for them.

Electric toothbrushes lose effectiveness if the users find the hand movements unfamiliar or are poorly motivated. Currently, a lot of research is being conducted on developing various electric-type oral care products. Excluding the use of auxiliary oral products such as dental floss and interdental toothbrushes, which are effective in managing the dental plaque between teeth [4], a manual toothbrush and a sonic vibrating toothbrush was used for the experimental group to clinically compare the effectiveness [5] of removing plaque from the teeth to help in choosing toothbrushes [6].

\section{Materials and Methods}

\section{Research subject}

\section{1) Participants}

A total of 60 volunteers with early gingivitis in their 30 s or older who voluntarily agreed to this study were included.

\section{2) Toothbrush used in the study}

The participants in the experimental group used a sonic electric toothbrush with 10-degree rotating bristles, and the participants in the control group used a manual toothbrush with double slim bristles.

\section{Method}

\section{1) Ethical approval}

This study was conducted according to the regulations of Dankook University's Institution Review Board and was approved under IRB File DKU2021-1-003.

\section{2) Tooth brushing education for participants}

To the experimental group, a sonic electric toothbrush with 10-degree rotating bristles was provided, and participants were instructed on how to brush the teeth as well as the tongue according to the guidelines. The control group was distributed with a double slim-bristle manual toothbrush, and the brushing method was instructed in the same way as the experimental group.

In the oral examination, the Simple Patient Hygiene Performance (S-PHP) index, gingivitis index, and halitosis were measured, and the values were recorded before the experiment, 1st week of the experiment, 2nd week of the experiment, and 4 th week of the experiment.

(1) Simple Patient Hygiene Performance index (S-PHP index)

The cotton pellet was wet with a disclosing solution, applied to the tooth surface, and then rinsed with water. The maxillary right first molar, maxillary right central incisor, maxillary left first molar, mandibular left first molar, mandibular left central incisor, and mandibular right first molar were studied, and whether the disclosing solution remained in the cervical mesial, distal, and incisal surfaces was checked. Each tooth was scored from 0 to a maximum of 5 points.

(2) Gingivitis index (GI index)

The gingivitis index was measured by dividing the gingival margin surrounding the teeth into four gingival margins (mesial, distal, buccal, and lingual).

(3) Halitosis measurement

Participants were told to collect gas in the mouth for about 3 minutes. They were observed so that they didn't open their mouth or talk. The tip of the halimeter was put into the oral cavity, and the gas was collected for 15 seconds. The measured values appearing on the monitor were recorded.

3) Data organization and statistical analysis

All statistical analyzes were performed using SPSS Statistics ver. 19.0 for Windows (SPSS Inc., Chicago, IL, USA), and all statistical significance levels were set to $\mathrm{p}=0.05$.

\section{Results}

\section{S-PHP index results}

It was found that there was an interaction effect between the two groups according to the period of toothbrush use. It was confirmed that the period of toothbrush use had an effect on the S-PHP index (Table 1).

\section{Gingivitis index changes}

The gingivitis index of the group using the toothbrush of the experimental group was found to decrease statistically significantly over the period of toothbrush use. The Gingivitis index also reduced over toothbrush use in the control group, but there was no statistically significant difference (Table 2). 
Table 1. S-PHP index results

\begin{tabular}{|c|c|c|c|c|c|c|}
\hline \multirow{2}{*}{ Time period } & Con. $(n=30)$ & Exp. $(n=30)$ & \multirow{2}{*}{$p$-value } & Within & Between & Interaction \\
\hline & \multicolumn{2}{|c|}{ Mean $\pm S D$} & & \multicolumn{3}{|c|}{$p$-value } \\
\hline Before & $3.03 \pm 0.48^{\mathrm{a}}$ & $3.03 \pm 0.44^{\mathrm{a}}$ & 0.960 & $<0.001^{* *}$ & $<0.001^{* *}$ & $<0.001^{* *}$ \\
\hline 1week & $3.01 \pm 0.44^{\mathrm{a}}$ & $2.67 \pm 0.53^{b}$ & 0.010 & & & \\
\hline 2weeks & $3.07 \pm 0.42^{\mathrm{a}}$ & $2.55 \pm 0.46^{b}$ & $<0.001$ & & & \\
\hline 4weeks & $3.10 \pm 0.55^{\mathrm{a}}$ & $2.31 \pm 0.49^{\mathrm{c}}$ & $<0.001$ & & & \\
\hline
\end{tabular}

Con.: control group, Exp.: experiment group, SD: standard deviation. "Compared between groups, p-value by Independent two-sample t-test. P: $\mathrm{p}$-value estimated by Repeated Measure ANOVA test. ${ }^{*} \mathrm{p}<0.05,{ }^{* *} \mathrm{p}<0.01$. ${ }^{\mathrm{abc}}$ Same letter means no statistical difference by Bonferroni's multiple comparison.

Table 2. Gingivitis index changes

\begin{tabular}{ccccccc}
\hline \multirow{2}{*}{ Time period } & \multicolumn{2}{c}{ Con. $(\mathrm{n}=30)$} & \multirow{2}{c}{ Exp. $(\mathrm{n}=30)$} & Within & Between & Interaction \\
\cline { 2 - 3 } & \multicolumn{2}{c}{ Mean \pm SD } & & & p-value \\
\hline Before & $1.14 \pm 0.27^{\mathrm{a}}$ & $1.13 \pm 0.28^{\mathrm{a}}$ & 0.926 & $0.000^{* *}$ & 0.167 & $0.002^{* *}$ \\
1 week & $1.10 \pm 0.29^{\mathrm{a}}$ & $1.08 \pm 0.25^{\mathrm{b}}$ & $0.703^{\#}$ & & \\
2weeks & $1.12 \pm 0.28^{\mathrm{a}}$ & $0.98 \pm 0.22^{\mathrm{c}}$ & $0.038^{\#}$ & & \\
4weeks & $1.08 \pm 0.31^{\mathrm{a}}$ & $0.90 \pm 0.18^{\mathrm{d}}$ & $0.008^{\#}$ & & \\
\hline
\end{tabular}

Con.: control group, Exp.: experiment group, SD: standard deviation. ${ }^{*}$ Compared between groups, $p$-value by Independent two-sample t-test. P: p-value estimated by Repeated Measure ANOVA test. ${ }^{*} \mathrm{p}<0.05,{ }^{* *} \mathrm{p}<0.01$. ${ }^{\mathrm{bc}}$ Same letter means no statistical difference by Bonferroni's multiple comparison.

Table 3. Halitosis measurement results

\begin{tabular}{|c|c|c|c|c|c|c|}
\hline \multirow{2}{*}{ Time Period } & Con. $(n=30)$ & Exp. $(n=30)$ & \multirow{2}{*}{$p$-value $e^{\#}$} & Within & Between & Interaction \\
\hline & \multicolumn{2}{|c|}{ Mean \pm SD } & & & p-value & \\
\hline Before & $66.23 \pm 11.46^{\mathrm{a}}$ & $67.03 \pm 12.15^{\mathrm{a}}$ & 0.794 & $0.002 * *$ & $0.013^{*}$ & $<0.001^{* *}$ \\
\hline 1week & $67.90 \pm 10.99^{a}$ & $64.70 \pm 12.44^{\mathrm{a}}$ & 0.295 & & & \\
\hline 2weeks & $66.77 \pm 8.17^{\mathrm{a}}$ & $58.97 \pm 11.71^{b}$ & 0.004 & & & \\
\hline 4weeks & $70.23 \pm 12.38^{\mathrm{a}}$ & $54.30 \pm 13.05^{b}$ & $<0.001$ & & & \\
\hline
\end{tabular}

Con.: control group, Exp.: experiment group, SD: standard deviation. ${ }^{\#}$ Compared between groups, p-value by Independent two-sample t-test. P: p-value estimated by Repeated Measure ANOVA test. ${ }^{*} \mathrm{p}<0.05,{ }^{* *} \mathrm{p}<0.01$. ${ }^{\mathrm{bc}}$ Same letter means no statistical difference by Bonferroni's multiple comparison.

\section{Halitosis measurement results}

It was found that there was an interaction effect between the two groups according to the period of toothbrush use. The period of toothbrush use had an effect on the halitosis measure (Table 3).

\section{Conclusion}

1. The S-PHP index of the group using the experimental group toothbrush showed a statistically significant decrease over the period of toothbrush use $(\mathrm{p}<0.001)$, and in the group using the control toothbrush, it decreased until 2 weeks after the experiment, and then increased again after 4 weeks.

2. As a result of analyzing the change in the gingivitis index, it was found that the gingivitis index of the group using the experimental group toothbrush decreased statistically significantly over the toothbrush use period. Although the gingivitis index also decreased in the control group over toothbrush use period, there was no statistically significant difference.

3. The concentration of halitosis in the group using the toothbrush of the experimental group was found to decrease statistically significantly over the period of toothbrush use $(p<0.001)$, and the concentration of halitosis in the group using the control toothbrush decreased until 2 weeks after the experiment and increased again after 4 weeks. 


\section{Conflict of Interest}

No potential conflict of interest relevant to this article was reported.

\section{ORCID}

Jin-Sil Kim, https://orcid.org/0000-0002-1569-4004

Chung-jae Lee, https://orcid.org/0000-0001-5648-0830

\section{References}

1. Paik DI, Kim HD, Jin BH, Park YD, Shin SC, Cho JW, et al. Clinical preventive dentistry. 5th ed. Seoul: Komoonsa; 2011:
65-114.

2. Loe $H$, Theilade E, Jensen SB. Experimental gingivitis in man. J Periodontol 1965;36:177-87.

3. Hickman J, Millett DT, Sander L, Brown E, Love J. Powered vs manual tooth brushing in fixed appliance patients: a short term randomized clinical trial. Angle Orthod 2002;72:135-40.

4. Lee DH, Shin SC. The study on the standardization of toothbrush design for recommended to individual dental patients. J Korean Acad Dent Health 1993;17:69-91.

5. Yang JS, Lim SA, Seong JH, Kim DK. A study on the elasticity loss of various toothbrushes. J Korean Acad Dent Health 2002; 26:341-60.

6. Ahn HW, Jwa SK, Ahn SH, An YH. A comparative appraisals for oral health education through to establishing the group toothbrushing facilities. Int J Clin Prev Dent 2010;6:35-45. 